\title{
Optimal First-Line Therapy for Acute Low-Tone Sensorineural Hearing Loss
}

\author{
Seung-Ho Shin ${ }^{1 *}$, Sung Wan Byun ${ }^{1 *}$, Sohl Park ${ }^{1}$, Eun Hye Kim ${ }^{1}$, Min Woo Kim², and Ho Yun Lee \\ ${ }^{1}$ Department of Otolaryngology-Head and Neck Surgery, College of Medicine, Ewha Womans University, Seoul, Korea \\ ${ }^{2}$ Department of Otorhinolaryngology, Daejeon Eulji University Medical Center, Eulji University School of Medicine, Daejeon, Korea
}

\begin{abstract}
Received April 14, 2021
Revised May 9, 2021

Accepted May 16, 2021
\end{abstract}

\section{Address for correspondence Ho Yun Lee, MD, PhD \\ Department of Otorhinolaryngology, Ewha Womans University \\ Mokdong Hospital, \\ 1071 Anyangcheon-ro, \\ Yangcheon-gu, Seoul 07985, Korea \\ Tel +82-2-2655-2011 \\ E-mail hoyun1004@gmail.com \\ *The first two authors contributed equally to this work.}

\begin{abstract}
Background and Objectives: We aimed to analyze treatment outcomes following different initial management approaches and confirm treatment regimens for acute low-tone sensorineural hearing loss (ALHL) that would yield the best results. Subjects and Methods: We retrospectively analyzed the medical records of 106 patients with ALHL who visited a university hospital's otology clinic from March 2013 to June 2019. Pure-tone averages at the initial visit and at 2 and 4 weeks after the initial visit were evaluated. Results: Forty-nine patients were enrolled in this study; of them, 41 (83.7\%) exhibited complete recovery (CR) at 2 weeks and $43(87.8 \%)$ exhibited CR at 1 month after the initial visit. Regression analysis revealed that CR at 2 weeks after the initial visit was associated with diuretic use [Exp(B): 10.309, 95\% confidence interval (Cl): 1.007-100]. An initial daily low-dose steroid use was marginally significant [Exp(B): 1.042, 95\% Cl: 0.997-1.092; $p=0.066]$. Isolated diuretic use [Exp(B): 25.641, 95\% $\mathrm{Cl}$ : $1.121-90.909 ; p=0.039]$ was an independent, good prognostic factor at 1 month after the initial visit. However, other treatment regimens did not affect the final results. Conclusions: A combination of initial daily administration of $\leq 30 \mathrm{mg}$ prednisolone plus diuretics was sufficient as the first-line treatment for ALHL. High-dose steroids and salvage intratympanic steroid injections can be applied as a second choice; however, the predicted outcome would not be good in that case.

J Audiol Otol 2021;25(4):209-216
\end{abstract}

Keywords: Hearing loss; Sudden; Meniere disease; Treatment outcome; Prognosis; Steroids.

\section{Introduction}

Acute low-tone sensorineural hearing loss (ALHL) without vertigo is a different inner ear disease from conventional sudden idiopathic sensorineural hearing loss (SSHL). It is more common in females in their 40s. Most patients have accompanying low-pitched tinnitus, aural fullness, and may experience a light dizzy sensation (not true vertigo) [1,2]. In addition, frequent spontaneous recovery and recurrence occurs and there is a high risk of progression into Meniere's disease (MD) [3].

The most used audiometric criteria for definite ALHL that was established by the Research Committee of the Ministry of Health, Labour and Welfare of Japan in 2011. It is as follows:

This is an Open Access article distributed under the terms of the Creative Commons Attribution Non-Commercial License (https://creativecommons.org/licenses/by-nc/4.0/) which permits unrestricted non-commercial use, distribution, and reproduction in any medium, provided the original work is properly cited.
1) sum of hearing thresholds at 125,250 , and $500 \mathrm{~Hz}$ of $\geq 70$ $\mathrm{dB}$ and 2) sum of hearing thresholds at 2,000, 4,000, and $8,000 \mathrm{~Hz}$ of $\leq 60 \mathrm{~dB}$. Cases that meet the audiometric criteria 1) with the same hearing levels at 2,000, 4,000, and $8,000 \mathrm{~Hz}$ in the contralateral ear are defined as probable ALHL [2]. ALHL is usually treated with combinations of steroids and diuretics compared with treatment for SSHL of oral steroids [2]. ALHL is assumed to be caused by an autoimmune response of endolymphatic sac and/or cochlear-specific endolymphatic hydrops $[1,4,5]$. Initial management is vital because the outcome after initial treatment of ALHL correlates with long-term results [6]. However, optimal treatment remains unknown, such as the optimal concentration of initial daily steroids or most effective type of diuretics, or the efficacy of intratympanic steroid (ITS) injection.

In this study, we aimed to analyze the treatment pattern and outcome following different initial management to confirm the best treatment regimen. 


\section{Subjects and Methods}

We retrospectively analyzed the medical records of 106 patients who visited an otology clinic at a Eulji University Hospital complaining of acute low-tone hearing loss from March 2013 to June 2019.

ALHL was defined by the following criteria: 1) a sensorineural hearing loss at low frequencies $(125,250$, and $500 \mathrm{~Hz})$, a sum of pure-tone thresholds at low frequencies of $\geq 70 \mathrm{~dB}$ and 2) a $\leq 60 \mathrm{~dB}$ sum of pure-tone thresholds at high frequencies $(2,4$, and $8 \mathrm{kHz}) .2)$ Intact tympanic membranes and 3) durations from symptom onset to treatment $\leq 14$ days. The following conditions were excluded: 1) previous history of cochlear symptoms such as tinnitus, aural fullness and 2) nystagmus observed at initial visit or accompanying vertigo.

Hearing thresholds of 125, 250, 500, 1,000, 2,000, 3,000, 4,000 , and $8,000 \mathrm{~Hz}$ were obtained, and the results of puretone audiometry performed at initial visit, 1 and 4 weeks after the initial visit were documented. The Institutional Review Board of the Eulji University Hospital approved this retrospective cohort study and waived the need to obtain written informed consent because of its retrospective nature (IRB number: 2020-12-002).

The study population comprised of 13 males and 36 females aged from 15-62 years, with a mean age of 41.96 years [standard deviation (SD): 12.56 years]. Mean duration from onset to treatment was 4.27 days (SD: 3.13 days, range: $0-14$ days). For accompanying symptoms, aural fullness was the most frequent $(91.8 \%, \mathrm{n}=45)$, followed by tinnitus $(55.1 \%, \mathrm{n}=27)$ and transient dizzy sensation $(28.6 \%, \mathrm{n}=14)$. The affected side was the right in $17(34.7 \%)$ and left in 32 (65.3\%) patients. For accompanying diseases, three patients had diabetes and four had hypertension.
The treatment regimen was chosen based on the preference and/or clinical experience of doctors. Basic concepts for prescribing steroids were as follows: most patients in whom steroids were not administered at local clinic were initially treated with low-dose steroids $[\leq 30 \mathrm{mg}$ of oral prednisolone (PD)]. If patients visited the local clinic complaining of persistent low-tone hearing loss, $60 \mathrm{mg}$ of oral PD was initially prescribed.

Oral PD was prescribed to $93.9 \%$ of patients $(n=46)$, with mean initial daily dosage of $27.96 \mathrm{mg}$ (SD: $18.141 \mathrm{mg}$, range: 10-60 mg, median: $30.00 \mathrm{mg}$ ) (Fig. 1). PD was initially prescribed twice daily for 7 days if the initial daily dose did not exceed $30 \mathrm{mg}$. If the initial daily dose of PD was $\geq 30 \mathrm{mg}$, the same dose of initial PD was administered once a day for 4 days and then tapered $10 \mathrm{mg}$ every 2 days.

Forty-five patients $(91.8 \%)$ were treated with diuretics. Of these, 34 patients were treated with $50 \mathrm{mg}$ of oral hydrochlorothiazide $(75.6 \%)$ for 4 weeks and six patients (13.3\%) with $70 \% 90 \mathrm{~mL}$ per day of isosorbide solution for 4 weeks and five patients $(11.1 \%)$ were treated with $50 \mathrm{mg}$ of oral spironolactone. For intratympanic dexamethasone injection, 18 patients $(36.7 \%)$ received four concomitant intratympanic dexamethasone injections in 2 weeks. Two patients were treated with ITSs plus diuretics.

Complete recovery (CR) was defined if the mean hearing threshold of lower three frequencies at 125,250 , and $500 \mathrm{~Hz}$ was $\leq 20 \mathrm{~dB}$.

To confirm which treatment modalities are effective, we performed a retrograde conditional logistic regression analysis of covariates that differed significantly by treatment outcome. This included use and types of diuretics, steroid dosage, and whether ITS was performed. All analyses were performed with the aid of IBM SPSS Statistics for Macintosh ver. 27.0

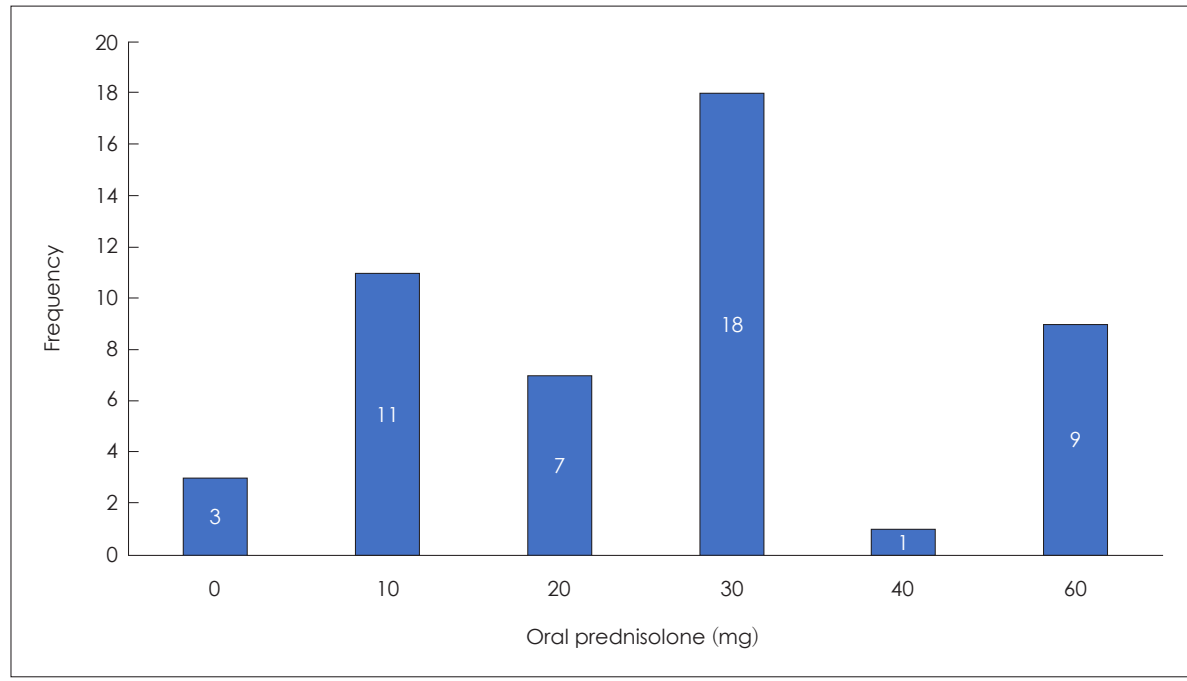

Fig. 1. Initial dosage of oral prednisolone. 
(IBM Corp., Armonk, NY, USA). $p$-values of $<0.05$ were considered statistically significant.

\section{Results}

Patient characteristics were summarized as Table 1. The mean hearing threshold was calculated from 500, 1,000, 2,000, and 3,000 Hz at initial visit was $15.995 \mathrm{~dB}$ (SD: 6.649). Mean hearing thresholds at 125, 250, and $500 \mathrm{~Hz}$ and 2,000, 4,000, and $8,000 \mathrm{~Hz}$ were $33.129 \mathrm{~dB}(\mathrm{SD}: 7.800 \mathrm{~dB})$ and $11.259 \mathrm{~dB}$ (SD: $5.177 \mathrm{~dB})$, respectively.

Forty-one patients $(83.7 \%)$ showed CR at 2 weeks after initial visit and 43 patients $(87.8 \%)$ showed a CR at 1 month later. Difference of CR at 2 week in accordance with initial treatment regimen is shown as Fig. 2. For hearing thresholds, low-tone, high-tone, and mean hearing thresholds at 2 week improved significantly compared to those evaluated at initial visit $(p<0.1)$ (Fig. 3). However, no significant differences were observed between hearing thresholds at 2 and 4 weeks.

Chi-square analysis was revealed that sex, affected side, accompanying symptoms, such as tinnitus, aural fullness, transient dizzy sensation, and accompanying diseases, including diabetes and hypertension did not differ according to recovery at 2 and 4 weeks $(p>0.05)$.

With regard to treatment modalities, whether diuretics were used or what kind of diuretics were applied, used initial daily dose of oral steroids, ITS were not different according to CR at 2 and 4 weeks $(p>0.05)$.

A retrograde conditional regression analysis with variables including initial steroid dosage, diuretics use and type, ITS,
Table 1. Patient characteristics

\begin{tabular}{|c|c|}
\hline Variables & Results \\
\hline Total & 49 \\
\hline Age (years) & $41.96 \pm 12.56$ \\
\hline \multicolumn{2}{|l|}{ Sex } \\
\hline Male & $13(26.5)$ \\
\hline Female & $36(73.5)$ \\
\hline \multicolumn{2}{|l|}{ Onset of treatment (days) } \\
\hline Mean $\pm S D$ & $4.27 \pm 3.13$ \\
\hline Range & $0-14$ \\
\hline \multicolumn{2}{|l|}{ Laterality of symptoms } \\
\hline Right & $17(34.7)$ \\
\hline Left & $32(65.3)$ \\
\hline \multicolumn{2}{|l|}{ Accompanying diseases } \\
\hline Diabetes mellitus & $3(6.1)$ \\
\hline Hypertension & $4(8.2)$ \\
\hline \multicolumn{2}{|l|}{ Accompanying symptoms } \\
\hline Tinnitus & $8(9.9)$ \\
\hline Aural fullness & $4(4.9)$ \\
\hline Transient dizzy sensation & $14(28.6)$ \\
\hline \multicolumn{2}{|c|}{ Hearing thresholds at initial visit } \\
\hline \multicolumn{2}{|c|}{ Hearing thresholds (dB) } \\
\hline Mean \pm SD & $15.995 \pm 6.649$ \\
\hline Range & $3.75-42.50$ \\
\hline \multicolumn{2}{|c|}{ Low-tone hearing thresholds (dB) } \\
\hline Mean $\pm S D$ & $33.129 \pm 7.800$ \\
\hline Range & $23.33-60.00$ \\
\hline \multicolumn{2}{|c|}{ High-tone hearing thresholds (dB) } \\
\hline Mean $\pm S D$ & $11.259 \pm 5.177$ \\
\hline Range & $1.67-20.00$ \\
\hline
\end{tabular}

Data are presented as $n(\%)$ or mean \pm SD unless otherwise indicated. SD: standard deviation

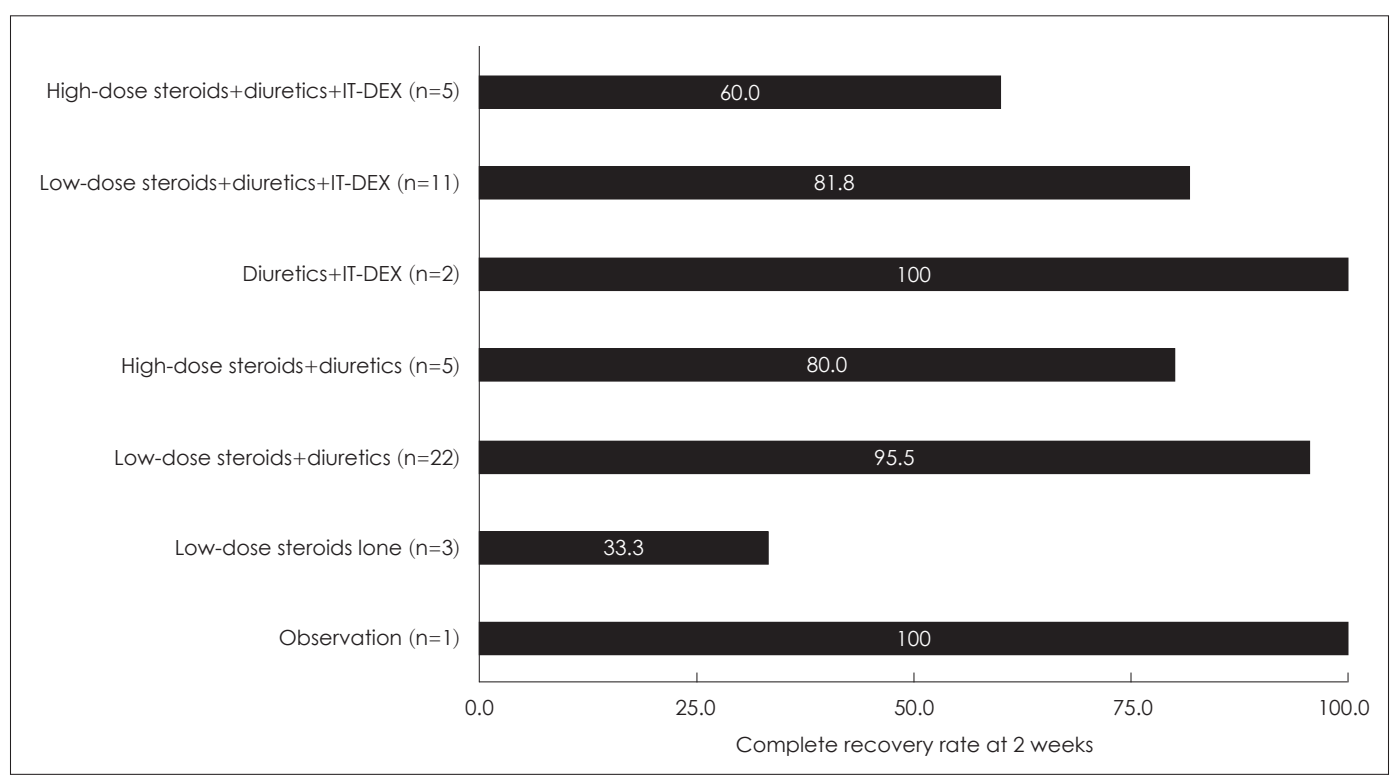

Fig. 2. Difference of complete recovery rate at 2 weeks in accordance with initial treatment regimens. IT-DEX: intratympanic dexamethasone. 


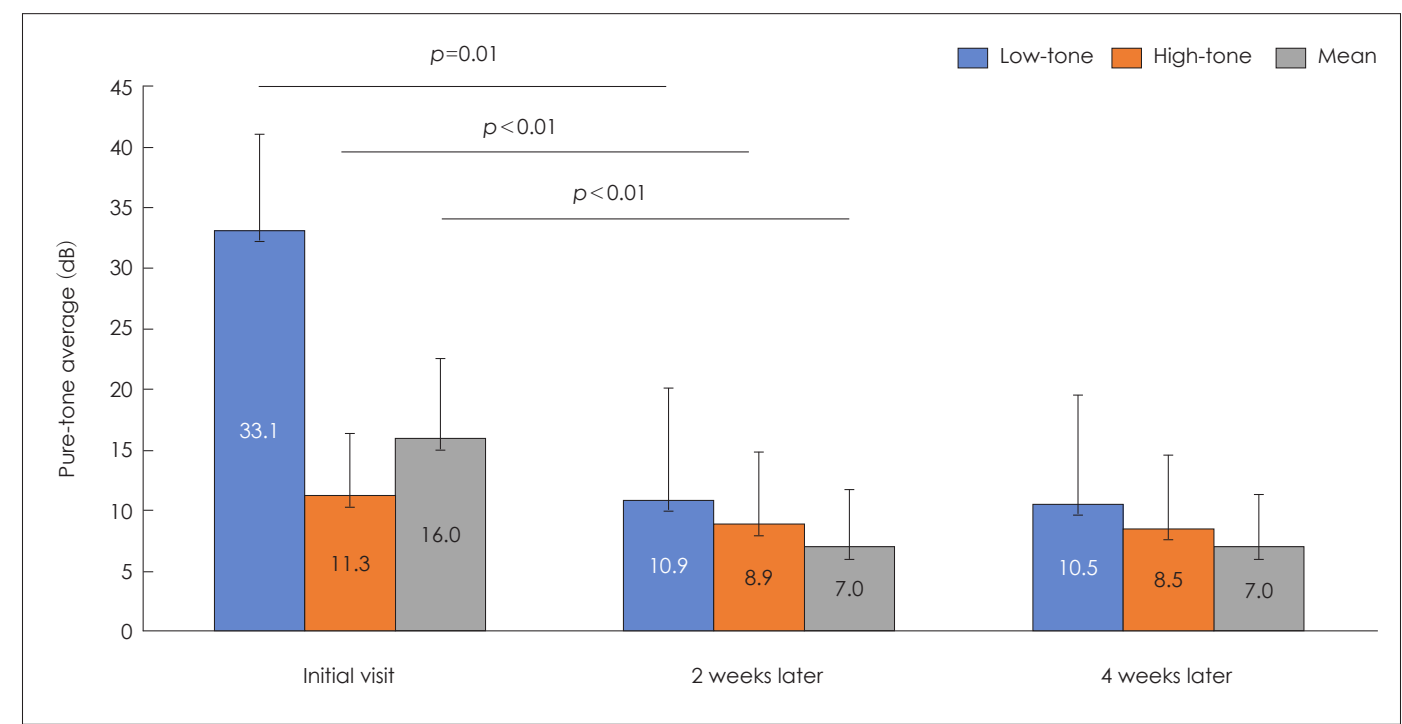

Fig. 3. Mean pure-tone average at each time.

and dizzy symptoms was performed to confirm the prognostic factors for treatment outcome. CR at 2 weeks was associated with diuretic use $[\operatorname{EXP}(B)=10.309,95 \%$ confidence interval $(C I)=1.007-100]$. An initial daily low-dose steroid use $[\operatorname{EXP}(\mathrm{B})=1.042,95 \% \mathrm{CI}=0.997-1.092, p=0.066]$ was marginally significant. Type of diuretics and ITS injection were not significant factors. For $\mathrm{CR}$ at 4 weeks, better recovery was associated with diuretic use $[\mathrm{EXP}(\mathrm{B})=25.641,95 \% \mathrm{CI}=$ 1.121-90.909, $p=0.039]$; however, other treatment regimens did not affect final results.

\section{Discussion}

In this study, we found that use of diuretics was significantly associated with better treatment outcomes at 2 weeks and 1 month later irrespective of type. Recent MD guideline recommends that diuretics may be offered to patients to reduce or prevent symptoms and disease attacks [7]. A systematic review on the use of diuretics for MD reported that vertigo was controlled and hearing improvements were achieved in 79\% and $42.1 \%$ patients, respectively [8].

Progression from ALHL to MD is a common; one study has reported that $27 \%$ of ALHL patients develop fluctuating hearing loss and $11 \%$ were finally diagnosed as classic MD [5]. Accompanying tinnitus and recurrent episodes of ALHL are contributing factors for developing MD from ALHL [9]. Similarly, $15.2 \%$ of ALHL patients have recurrence within a 1 year after initial outbreak and outcome after initial treatment is correlated with long-term results [6].

We found that the type of diuretics administered did not affect treatment outcome. Our systematic review showed that the most commonly used diuretics for treatment of ALHL were thiazide diuretics (Table 2). The most common complications of thiazide include dose-dependent metabolic imbalance such as hypokalemia, hypomagnesemia, hyperuricemia, decreased urinary calcium excretion, and glucose intolerance [10]. However, these can be minimized by using lower dose [10]. An oral osmotic diuretic, isosorbide solution, is an alternative. It is well tolerated for most of patients with MD with only a few experiencing headaches or nausea after medication [11]. We hypothesize that changing the type of diuretics is a valid option if complications occur after initial diuretic administration. It is not a priority to change diuretic for better treatment outcomes because type of diuretics was not chosen in our regression model. However, an additional study with more larger sample size is necessary to confirm this.

We found that ALHL patients who were responsive to lowdose steroids tended to have better recovery at 2 weeks after initial treatment, though this was marginally significant. In a meta-analysis, no significant difference in recovery was observed between steroid treatment and diuretic treatment, implying that both are equally effective [12].

In general, there is a belief that a higher dose of steroids may be more effective than a lower dose or where there is no improvement after initial treatment. Consistent with this, an early study dealing with ALHL reported that some patients who did not respond to an initial $60 \mathrm{mg}$ intravenous and oral PD recovered after $200 \mathrm{mg}$ intravenous PD, advocating use of higher dose steroids in ALHL [13]. Similarly, corticosteroids can be effective at high doses within 7 days if initial treatment if the low doses fails to reduce symptoms [14].

Interestingly, we found a tendency for better outcome at 


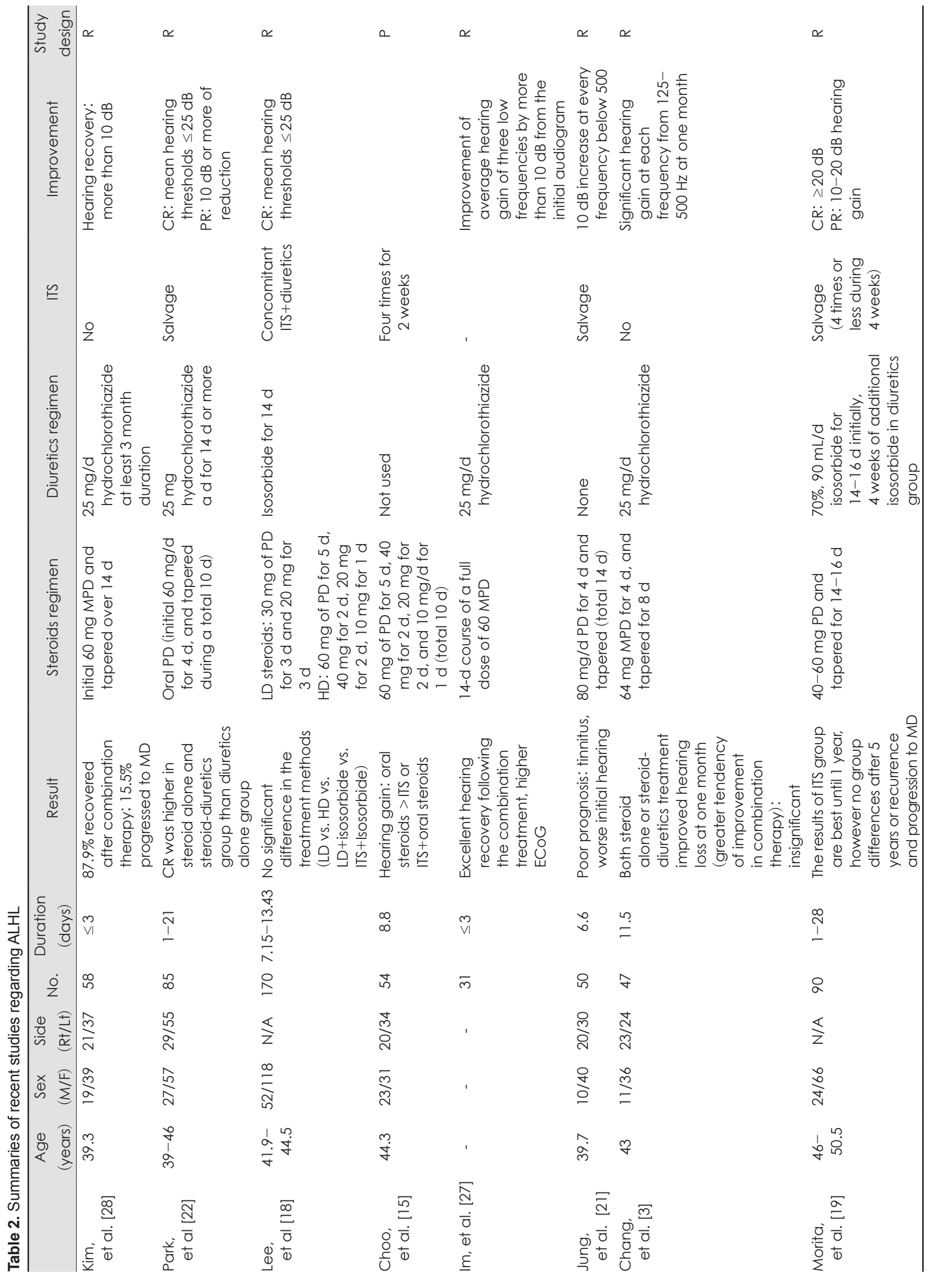




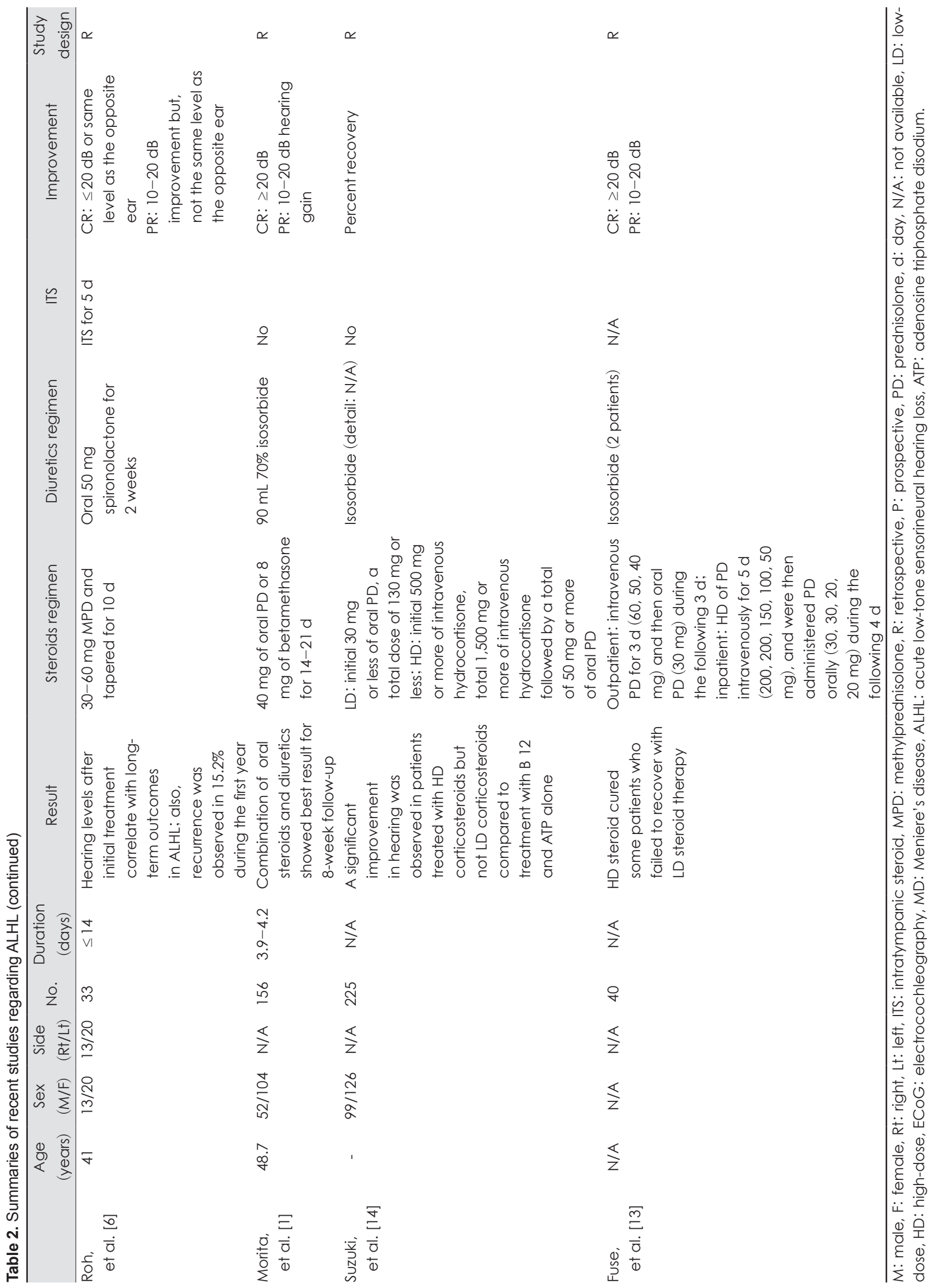


2 weeks in patients who were treated initially with low-dose steroids. Therefore, low-dose steroids were sufficient for patients who are responsive to steroid treatment. Considering the mean or median value of steroid doses, an initial daily dose under $30 \mathrm{mg}$ can be defined as a low dose. Due to the characteristics of the university referral hospital and retrospective nature of this study, patients who were less responsive to lowdose steroid treatment at the local clinic might be commonly treated with high-dose steroids in out clinic and they might be also less responsive to high-dose steroids.

A recent prospective study reported that the treatment effect of oral steroids alone (starting with $60 \mathrm{mg}$ of oral PD) was significantly better than ITS as an initial treatment or oral steroids plus concurrent ITS [15]. In fact, their inclusion criteria were somewhat different from other ALHL studies; therefore, their result was more closely related to idiopathic sudden hearing loss itself because they defined low-frequency hearing loss as $20 \mathrm{~dB}$ or more of hearing loss at 250,500 , and $1,000 \mathrm{~Hz}$ (1,000 Hz was included but $125 \mathrm{~Hz}$ was not included).

Similarly, a Japanese group compared definite ALHL and sudden low-tone loss that does not meet the criteria of ALHL [16]. As a result, poor recovery and a tendency for more common recurrence was observed at 1 year in the sudden lowtone loss group; however, it was statistically insignificant. In their study, they also analyzed progression into MD with electrocochleography and electronystagmography [16]. As a result, they argued that the current widely used inclusion criteria for ALHL may need to be complemented by additional electronystagmography and electrocochleography.

It was previously reported that ITS yielded a CR in most patients with ALHL [17]. Partially consistent with our finding, a comparative study with a larger sample size reported no difference in treatment outcome between low dose steroids, high dose steroids, low dose steroids plus diuretics, and ITS plus diuretics [18]. In contrast, ITS as a salvage treatment after 2 weeks of combined steroid-diuretic treatment yields better outcome than observation or continuous diuretics treatment alone for 1-year follow-up, though final treatment outcome after 5 years was not different, irrespective of treatment strategies [19]. We did not find any beneficial effect of concurrent use of ITS; however, there is some scope to adopt ITS as a salvage treatment.

A Korean group has compared steroid treatment alone with combination treatments [3]. Both treatment regimens improved hearing; however, there was a greater tendency for improvement in combination treatment but this was statistically insignificant.

Aside from treatment regimens, many prognostic factors have been revealed. Younger age, low-grade severity of ini- tial hearing loss, early treatment, and female are good prognostic factors [20]. On the other hand, accompanying tinnitus, occurrence of vertigo, bilateral involvement, longer symptom duration, and $1 \mathrm{kHz}$ involvement are poor prognostic factors $[18,21,22]$.

Of the patients who showed final CR, two patients (a 60-yearold female and a 54-year-old male patient) showed a delayed recovery compared to other patients. They were treated with high-dose steroids plus hydrochlorothiazide and low-dose steroids plus hydrochlorothiazide and concomitant ITS, respectively. Their mean low-tone hearing thresholds at initial assessment were 30 and $60 \mathrm{~dB}$ and improved to 25 and $21.67 \mathrm{~dB}$ at 2 week, respectively. Therefore, we assumed that the individual difference in response to treatment might account for the delayed recovery more than expected, and more severe impairment may need more time for recovery.

Magnetic resonance imaging (MRI) has been investigated to reveal the pathogenesis of ALHL. In a 3T three-dimensional fluid attenuated inversion recovery study, cochlear endolymphatic hydrops was observed in $88.9 \%$ of ALHL patients who met the criteria of definite ATHL. Interestingly, vestibular endolymphatic hydrops were also found in these patients, though they had no vertigo, but some of them (36\%) had subtle dizzy symptoms. These findings were similar to MD [23]. These findings showed an increased volume of endolymphatic space/total fluid space in the vestibule in the recurrence group. Increased endolymphatic volume in the cochlea was observed in no cure group, suggesting that MRI can predict the prognosis of ALHL [24].

Vestibular evoked myogenic potentials (VEMPs) have been used to predict the progression into MD or treatment outcome. Higher abnormal VEMPs suggestive of saccular hydrops are observed in MD compared with ALHL [25]. Combining cVEMP and galvanic stimulation can predict final recovery more precisely by revealing the extent of saccular dysfunction [26].

Taken together, our findings should be cautiously interpreted. We assume that patients who are unresponsive to low-dose steroids tend to have no or little response to higher steroids. Different from general sudden idiopathic hearing loss, initial low-dose steroids can be considered as a first-line treatment for ALHL patients in an out-patient clinic instead of high-dose steroid use. Diuretics should be used from the beginning of treatment. ITS as a salvage treatment may be considered.

Table 2 summarizes the characteristics of recent ALHL studies focusing on treatment outcome from 2002 to 2020 [1,3, $6,13,14,15,18,19,22,27,28]$. Enrollment design, initial daily steroid dose, diuretics usage, ITS protocol, criteria for recovery varied widely from studies. For appropriate comparison, these should be unified first and then a prospective random- 
ized controlled trial should be performed to determine the optimal treatment regimen.

In conclusion, combination of low-dose steroids under $30 \mathrm{mg}$ of PD plus diuretics was sufficient for the first line treatment for ALHL. High-dose steroids and salvage ITS can be used a second choice treatment; however, the predictable outcome cannot be confirmed. Clinicians should counsel patients on these negative predictable results before the initiation of salvage treatment.

\section{Acknowledgments}

This research was supported by by the Basic Science Research Program through the National Research Foundation of Korea (NRF), funded by the Ministry of Education (NRF-2017R1C1B5017839 for HYL).

\section{Conflicts of interest}

The authors have no financial conflicts of interest.

\section{Author Contributions}

Conceptualization: Seung-Ho Shin and Ho Yun Lee. Data curation: Sohl Park and Min Woo Kim. Formal analysis: Ho Yun Lee. Methodology: Ho Yun Lee. Resources: Sung Wan Byun and Eun Hye Kim. Software: Sung Wan Byun. Supervision: Seung-Ho Shin and Sung Wan Byun. Validation: Sung Wan Byun. Visualization: Ho Yun Lee. Writing - original draft: Seung-Ho Shin and Ho Yun Lee. Writing - review \& editing: Seung-Ho Shin and Sung Wan Byun. Approval of final manuscript: all authors.

\section{ORCID iDs}

Seung-Ho Shin

Sung Wan Byun

Sohl Park

Eun Hye Kim

Min Woo Kim

Ho Yun Lee https://orcid.org/0000-0001-8093-2673 https://orcid.org/0000-0002-5458-6401 https://orcid.org/0000-0002-1743-7044 https://orcid.org/0000-0002-3932-3056 https://orcid.org/0000-0002-4025-6305 https://orcid.org/0000-0002-9590-3477

\section{REFERENCES}

1) Morita S, Suzuki M, Iizuka K. A comparison of the short-term outcome in patients with acute low-tone sensorineural hearing loss. ORL J Otorhinolaryngol Relat Spec 2010;72:295-9.

2) Yoshida T, Sone M, Kitoh R, Nishio SY, Ogawa K, Kanzaki S, et al. Idiopathic sudden sensorineural hearing loss and acute low-tone sensorineural hearing loss: a comparison of the results of a nationwide epidemiological survey in Japan. Acta Otolaryngol 2017;137:S38-43.

3) Chang J, Yum G, Im HY, Jung JY, Rah YC, Choi J. Short-term outcomes of acute low-tone sensorineural hearing loss according to treatment modality. J Audiol Otol 2016;20:47-52.

4) Junicho M, Aso S, Fujisaka M, Watanabe Y. Prognosis of low-tone sudden deafness-does it inevitably progress to Meniere's disease? Acta Otolaryngol 2008;128:304-8.

5) Yamasoba T, Kikuchi S, Sugasawa M, Yagi M, Harada T. Acute low-tone sensorineural hearing loss without vertigo. Arch Otolaryngol Head Neck Surg 1994;120:532-5.

6) Roh KJ, Lee EJ, Park AY, Choi BI, Son EJ. Long-term outcomes of acute low-tone hearing loss. J Audiol Otol 2015;19:74-8.

7) Basura GJ, Adams ME, Monfared A, Schwartz SR, Antonelli PJ, Burkard R, et al. Clinical practice guideline: Ménière's disease exec- utive summary. Otolaryngol Head Neck Surg 2020;162:415-34.

8) Crowson MG, Patki A, Tucci DL. A systematic review of diuretics in the medical management of Ménière's disease. Otolaryngol Head Neck Surg 2016;154:824-34.

9) Stölzel K, Droste J, Voß LJ, Olze H, Szczepek AJ. Comorbid symptoms occurring during acute low-tone hearing loss (AHLH) as potential predictors of Menière's disease. Front Neurol 2018;9:884.

10) Palmer BF, Naderi AS. Metabolic complications associated with use of thiazide diuretics. J Am Soc Hypertens 2007;1:381-92.

11) Lee JD, Kim HJ, Jung J, Kim SH, Kim BG, Kim KS. Is dehydration test using isosorbide useful in Meniere's disease? Acta Otolaryngol 2016;136:1107-9.

12) Zhu Y, Li G, Zhuang H, Yang Z, Sun J, Xiong G, et al. Meta-analysis comparing steroids and diuretics in the treatment of acute low-tone sensorineural hearing loss. Ear Nose Throat J 2021;100:281S-5S.

13) Fuse T, Aoyagi M, Funakubo T, Sakakibara A, Yoshida S. Shortterm outcome and prognosis of acute low-tone sensorineural hearing loss by administration of steroid. ORL J Otorhinolaryngol Relat Spec 2002;64:6-10.

14) Suzuki M, Otake R, Kashio A. Effect of corticosteroids or diuretics in low-tone sensorineural hearing loss. ORL J Otorhinolaryngol Relat Spec 2006;68:170-6.

15) Choo OS, Yang SM, Park HY, Lee JB, Jang JH, Choi SJ, et al. Differences in clinical characteristics and prognosis of sudden low- and high-frequency hearing loss. Laryngoscope 2017;127:1878-84.

16) Fushiki H, Junicho M, Kanazawa Y, Aso S, Watanabe Y. Prognosis of sudden low-tone loss other than acute low-tone sensorineural hearing loss. Acta Otolaryngol 2010;130:559-64.

17) Alatas N. Use of intratympanic dexamethasone for the therapy of low frequency hearing loss. Eur Arch Otorhinolaryngol 2009;266: 1205-12.

18) Lee CK, Lee JB, Park KH, Lee HY, Choi MJ, Ban W, et al. Significance of $1 \mathrm{kHz}$ pure-tone threshold in acute low-frequency sensorineural hearing loss. Otol Neurotol 2018;39:e950-5.

19) Morita S, Nakamaru Y, Fujiwara K, Iizuka K, Masuya M, Homma A, et al. The short-and long-term outcome of intratympanic steroid therapy as a salvage treatment for acute low-tone sensorineural hearing loss without episodes of vertigo. Audiol Neurootol 2016;21:132-40.

20) Sato H, Kuwashima S, Nishio SY, Kitoh R, Fukuda S, Hara A, et al. Epidemiological survey of acute low-tone sensorineural hearing loss. Acta Otolaryngol 2017;137:S34-7.

21) Jung AR, Kim MG, Kim SS, Kim SH, Yeo SG. Clinical characteristics and prognosis of low frequency sensorineural hearing loss without vertigo. Acta Otolaryngol 2016;136:159-63.

22) Park MJ, Kim SH, Kim SS, Yeo SG. Clinical characteristics and short-term outcomes of acute low frequency sensorineural hearing loss with vertigo. Clin Exp Otorhinolaryngol 2018;11:96-101.

23) Shimono M, Teranishi M, Yoshida T, Kato M, Sano R, Otake H, et al. Endolymphatic hydrops revealed by magnetic resonance imaging in patients with acute low-tone sensorineural hearing loss. Otol Neurotol 2013;34:1241-6.

24) Inui $H$, Sakamoto $T$, Ito $T$, Kitahara T. Magnetic resonance imaging of the endolymphatic space in patients with acute low-tone sensorineural hearing loss. Auris Nasus Larynx 2019;46:859-65.

25) Wu CL, Young YH. Vestibular evoked myogenic potentials in acute low-tone sensorineural hearing loss. Laryngoscope 2004;114:2172-5.

26) Wang CT, Fang KM, Young YH, Cheng PW. Vestibular-evoked myogenic potential in the prediction of recovery from acute low-tone sensorineural hearing loss. Ear Hear 2010;31:289-95.

27) Im GJ, Kim SK, Choi J, Song JJ, Chae SW, Jung HH. Analysis of audio-vestibular assessment in acute low-tone hearing loss. Acta Otolaryngol 2016;136:649-54.

28) Kim H, Lee H, Kim YC, Park E, Choi J, Rah YC, et al. Update on clinical manifestation in acute low-tone hearing loss. Korean J Otorhinolaryngol-Head Neck Surg 2020;63:403-8. 